\title{
Characterization and Divergence Analysis of Duplicated R2R3-MYB Genes in Watermelon
}

\author{
Jin Wang \\ Key Laboratory of Horticultural Plant Biology (MOE), College of Horticulture and Forestry Sciences, \\ Huazhong Agricultural University, Wuhan 430070, China; and College of Horticulture, Nanjing \\ Agricultural University, Nanjing 210000, China \\ Yue Liu, Xueliang Chen, and Qiusheng Kong \\ Key Laboratory of Horticultural Plant Biology (MOE), College of Horticulture and Forestry Sciences, \\ Huazhong Agricultural University, Wuhan 430070, China
}

\begin{abstract}
AdDitional INDEX words. Citrullus lanatus, duplicated gene, expression pattern, fruit development, neo-functionalization
Abstract. Watermelon (Citrullus lanatus) is an economically important cucurbit (Cucurbitaceae) crop cultivated globally for its nutritional benefits. Fruit development in watermelon is characterized by fast fruit expansion attributed to unique biological processes. Myeloblastosis $(M Y B)$ family genes play important roles in plant growth and development, especially $R 2 R 3-M Y B$-type genes. However, the evolution of $R 2 R 3-M Y B$ family genes in the watermelon genome and whether they participate in the regulation of watermelon fruit development remain unknown. To address these questions, duplication modes of $R 2 R 3-M Y B$ family genes were identified and their expression profiles were investigated during watermelon fruit development. A total of 48 duplicated gene pairs were identified among the 89 $R 2 R 3-M Y B s$ in watermelon. Segmental and transposed duplication events play major roles in the $R 2 R 3-M Y B$ family gene expansion process. The ratio of nonsynonymous mutation and synonymous mutation analysis indicated that all the duplicated $R 2 R 3-M Y B$ s experienced negative selection. Gene structures and cis-element compositions in promoter sequences exhibited abundant divergences between the $R 2 R 3-M Y B$ duplicated genes. Transcriptome analyses of seed, rind, and flesh during fruit development showed that only two duplicated gene pairs had significantly similar expression patterns, whereas divergent expression profiles were found between the remaining duplicated gene pairs. Tissue-specific and development stage-specific divergent expression patterns demonstrated that neo-functionalization occurred between watermelon $R 2 R 3-M Y B$ duplicated genes. The current study provides valuable information for further functional analyses of $R 2 R 3-M Y B$ s in watermelon.
\end{abstract}

Transcription factors (TFs) are extensively involved in the regulation of plant growth and development. $M Y B$ is an important TF gene family with the largest members (Dubos et al., 2010). Its N-terminal region has a highly conserved DNA binding domain, which is called MYB domain. A MYB gene usually contains one to four incomplete MYB domain repeats $(\mathrm{R})$ of $\approx 52$ amino acid residues. Based on the number of MYB repeats, the $M Y B$ family gene can be divided into four types, namely, $4 R-M Y B$-type with four repeats, $3 R-M Y B$-type with three repeats, $R 2 R 3-M Y B$-type with two repeats, and $M Y B$ related-type with a single repeat or partial $M Y B$-related repeats (Jia et al., 2004). R2R3-MYB is dominant and has the largest members in most plants (Rosinski and Atchley, 1998), and it has a wide range of functions, including the regulation of plant growth and development, primary and secondary metabolism, and response to biotic and abiotic stresses (Stracke et al., 2001).

Gene duplication has an important role in the expansion of the gene family and functional diversity. Large-scale gene duplication and single-gene duplication are two types of gene duplication models that widely exist in eukaryotic genomes (Wang et al., 2013b). Large-scale gene duplication includes whole-genome duplication (WGD) and segmental duplication (Salman-Minkov et al., 2016). Single-gene duplication is also

Received for publication 30 Oct. 2019. Accepted for publication 18 Dec. 2019. Published online 8 July 2020.

Q.K. is the corresponding author: E-mail: qskong@mail.hzau.edu.cn.

This is an open access article distributed under the CC BY-NC-ND license (https://creativecommons.org/licenses/by-nc-nd/4.0/). prevalent in plant genomes and consists of three duplication models, including transposed duplication, proximal duplication, and tandem duplication (Freeling, 2009). Transposed duplication generates a duplicated gene on a new chromosome position via replicative transposition (Panchy et al., 2016). Tandem duplication results in a gene pair produced by an unequal cross between alleles (Hahn, 2009). The proximal gene pairs are two duplicated genes separated by a small number of genes (Wang et al., 2012). The WGD in watermelon (Citrullus lanatus) has seven major triplications dating back to between 76 and 130 million years ago and generated 302 paralogous relationships covering $29 \%$ of the genome (Guo et al., 2013). The segmental duplication event reportedly has a major role in the expansion of the $M Y B$ gene family in pineapple (Ananas comosus) (Liu et al., 2017).

After duplication, mutations increasingly accumulate in the regulatory and coding regions of duplicated genes during the course of evolution (Zhu et al., 2014). Different regulatory regions may lead to changes in expression levels, and different coding regions may result in new functions (Long and Thornton, 2001). The function-differentiated duplicated genes may undergo positive or negative selection. The ratio of nonsynonymous mutation $(\mathrm{Ka})$ to synonymous mutation $(\mathrm{Ks})$ is used to estimate the selection pressure of duplicated genes (Zhang et al., 2006). Many evolutionary models have been proposed to elucidate the gene status after duplication, including the shortand long-term retention or loss function (Qiao et al., 2018). Furthermore, three functional models are used to describe the 
functions of the retained duplicated genes, including gene dosage, sub-functionalization, and neo-functionalization (Flagel and Wendel, 2009). Gene dosage is one mechanism that affects the functions by changing the genes number of copies after duplication, and recent research has suggested that the presence of gene dosage may be beneficial to gene duplication (Panchy et al., 2016). The sub-functionalization model suggests that each duplicated gene retains its original ancestral function during the natural mutational process (Freeling et al., 2015). Sub-functionalization was found between the duplicated $M Y B$ genes ScAN2 and ScAN1 in potato (Solanum commersonii) in the response to cold stress (D'Amelia et al., 2018). The phenomenon that one duplicated gene keeps the ancestral function while the other gene acquires a new function is called neo-functionalization (Duarte et al., 2006). Neo-functionalization has been reported for the B-class MADS-box duplicated genes of PFGLO1 and PFDEF in Physalis floridana (Zhang et al., 2015).

Watermelon is an economically important cucurbit crop widely grown for its large, edible fruit. The watermelon planted area and total yield are ranked among the world's top 10 for fresh fruits (Food and Agriculture Organization of the United Nations, 2018). Because a whole genome sequence of the crop is available, watermelon has become a crop widely researched by plant breeders and plant physiologists. A total of 162 MYB genes have been identified in the watermelon genome, and expression patterns of $25 M Y B$ genes were measured in watermelon seedlings under abiotic stresses (Xu et al., 2018). This study provides a fundamental understanding of the possible roles of $M Y B$ family genes in watermelon stress tolerance. Fruit development is one of the most important biological processes of watermelon; it determines the economic value of watermelon production. $M Y B$ genes have been reported to widely participate in the regulation of fruit development (Machemer et al., 2011). However, the roles $R 2 R 3-M Y B$ genes in regulating watermelon fruit development and whether the functions of duplicated $R 2 R 3-M Y B$ genes are different during fruit development are still unknown.

In this study, duplicated $R 2 R 3-M Y B$ gene pairs were identified in the watermelon genome. Their expression profiles in rind, flesh, and seeds were determined during fruit development. Expression patterns of duplicated genes were associated with their $\mathrm{Ka} / \mathrm{Ks}$ values, gene structures, and cis-element compositions. This study aimed to identify the $R 2 R 3-M Y B$ genes involved in watermelon fruit development and clarify the function divergences of duplicated $R 2 R 3-M Y B$ genes.

\section{Materials and Methods}

IDENTIFICATION OF R2R3-MYB GENES. The genome of watermelon cultivar 97103 (v1) was used as the reference genome (Guo et al., 2013). All protein sequences and coding sequences were downloaded from the Cucurbit Genomics Database (Zheng et al., 2019). The hidden Markov model (HMM) profile of the MYB DNA-binding domain (PF00249) was downloaded from the Pfam database (El-Gebali et al., 2019). HMMER (Finn et al., 2011) was applied to identify the $M Y B$ family members with a cutoff of $\mathrm{E} \leq 0.01$. Protein domains of R2R3-MYB were validated by SMART (Letunic and Bork, 2018). Gene structures were analyzed using the Gene Structure Display Server (Hu et al., 2015). The MEME online tool (version 5.1.0; National Institutes of Health, Bethesda,
MD) was used to investigate conserved domains. Weblogo (Crooks et al., 2004) was used to draw the sequence logos. Protein modeling was predicted using SWISS-MODEL (Waterhouse et al., 2018).

IDENTIFICATION OF DUPLICATED GENES AND CIS-ELEMENT ANALYSIS. MCScanX-transposed was used to identify and classify gene duplication modes (Wang et al., 2013a). Circos (Krzywinski et al., 2009) was used to display the synteny relationships. KaKs_Calculator1.2 (Zhang et al., 2006) was applied to estimate the $\mathrm{Ks}$ and $\mathrm{Ka}$ values. Bedtools (Quinlan and Hall, 2010) was used to obtain the $1-\mathrm{kb}$ upstream sequence for each gene. Cis-elements were predicted using PlantCARE (Lescot et al., 2002). The cis-elements index value of the duplicated genes were calculated using the following equation: [1-(common cis-elements/all cis-elements)]. This is used to measure the dissimilarity of cis-elements between two duplicated genes. The value of the cis-element index ranged from 0 to 1 , and the larger value indicated greater divergence.

Transcriptome Data analysis. The transcriptome data of watermelon seeds sampled at 25, 31, 37, and $49 \mathrm{~d}$ after pollination (DAP) under BioProject ID PRJNA319011 (University of Nebraska, Lincoln, NE) and transcriptome data of watermelon fruit flesh and rind sampled at 10, 18, 26, and 34 DAP under BioProject ID SRP012849 (Boyce Thompson Institute, Ithaca, NY) were downloaded from the National Center for Biotechnology Information Sequence Read Archive (Bethesda, MD) database. All the annotated gene sequences of the watermelon cultivar 97103 reference genome were used as the reference transcriptome. Kallisto (Bray et al., 2016) was used to calculate the transcript per million (TPM) value for each gene. RStudio (version 1.0.136; RStudio, Boston, MA) was used to conduct the data analysis and draw the plots.

\section{Results}

IDENTIFICATION OF WATERMELON R2R3-MYB GENES. The HMM profile of MYB was used to search for watermelon genome-wide annotated proteins; $164 M Y B$ genes were identified. These $M Y B$ genes were further validated and classified by searching both the Pfam and SMART databases, resulting in 89 $R 2 R 3$-type genes, $5 R 3$-type genes, and $70 M Y B$-related-type genes. No $R 4$-type genes were found in the watermelon genome. The $R 2 R 3$-type genes were selected for further analysis and named $C l R 2 R 3-M Y B$. Information regarding these genes is listed in Supplemental Table 1. Sequence logo was used to exhibit the characteristics of $C l R 2 R 3-M Y B$ conserved motifs (Fig. 1). Results revealed 53 amino acid residues in the $\mathrm{R} 2$ repeat and 71 amino acid residues in the R3 repeat. Highly conserved Trp residues were found on positions 6, 26, and 46 of the R2 repeat and 25, 45, 64 on the $\mathrm{R} 3$ repeat, respectively. The threedimensional protein structure models showed that each of the two domains build an HTH structure, which is the main characterization of $R 2 R 3-M Y B$ family genes. Amino acids such as Glu-10, Gly-22, Arg-37, Lys-40, Ser-41, Lys-45, Cys-42, Leu44, Asn-48, and Leu-50 in the R2 repeat and Glu-10, Ala-29, Thr-56, Asp-57, Asn-58, Lys-61, and Asn-62 in the R3 repeat were also conserved among watermelon $R 2 R 3-M Y B$ genes.

Duplication events of ClR2R3-MYB Genes. A total of 48 ClR2R3-MYB duplicated gene pairs were identified, including 27 segmental duplicated pairs, 19 transposed duplicated pairs, 1 proximal duplicated pair, and 1 tandem duplicated pair (Supplemental Table 2). Duplicated gene pairs and their 
genome locations are displayed in the Circos plot (Fig. 2). The duplicated genes were distributed on all 11 watermelon chromosomes and tended to cluster at the two ends of each chromosome. Chromosome 5 harbored the largest number of
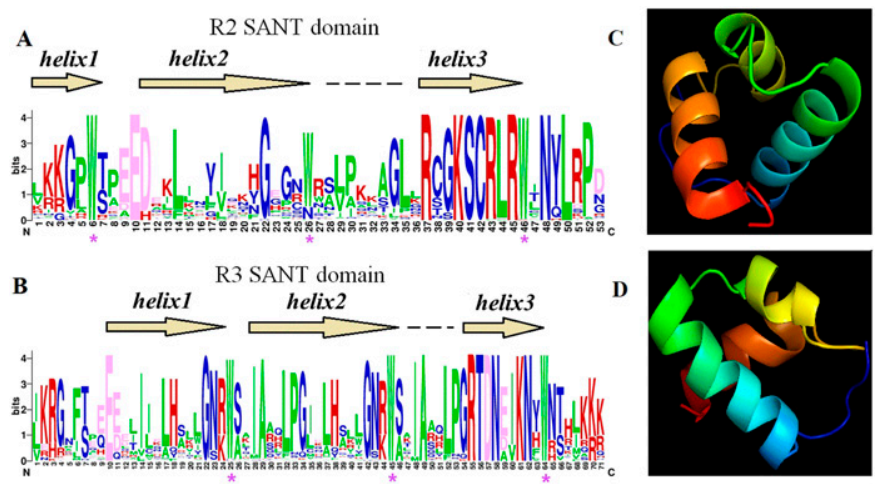

Fig. 1. Domains of $C l R 2 R 3-M Y B$ family genes and protein three-dimensional (3D) structure models of R2 and R3 MYB repeats. (A) R2 domain. (B) R3 domain. The bit score indicates the information content for each position in the sequence and the purple asterisks indicate the conserved tryptophan residues (Trp, W). (C) $\mathrm{R} 2$ repeats 3D structure model. (D) R3 repeats 3D structure models. duplicated genes. Specially, mutually segmental duplicated events were found among the triple genes of Cla013979Cla020663-Cla022601, Cla005982-Cla007586-Cla021520, Cla010332-Cla019115-Cla018610, and Cla007715Cla019451-Cla001341.

Gene STRUCTURE divergences OF DUPLICATEd CLR2R3MYB GENES. To compare gene structures of duplicated genes, DNA and mRNA sequences of each gene were aligned to analyze the exon-intron composition (Fig. 3). Exons ranged from one to four in the duplicated genes. The duplicated gene pair Cla017337-Cla015165 had the highest number of exons, whereas duplicated gene pairs Cla020702-Cla022542, Cla020633-Cla022601, Cla004362-Cla013979, Cla013979Cla020633, and Cla013979-Cla022601 harbored only one exon in each gene. A total of 36 duplicated gene pairs comprised similar exon-intron compositions. The remaining 12 duplication gene pairs had different gene structures, including three segmental duplicated gene pairs and nine transposed duplicated gene pairs. The different gene structures probably lead to functional divergences between the duplicated genes.

Cis-elements divergences OF DUPLiCATEd CLR2R3-MYB GENES. To compare the cis-regulatory element composition in the promoter region between the duplicated gene pair, a $1-\mathrm{kb}$ upstream sequence of each gene was selected and the putative cis-regulatory elements were identified (Supplemental Table 3). To quantify the dissimilarity of cis-element composition between duplicated genes, the cis-element index was calculated for each gene pair. The density distribution of the cis-element index for segmental and transposed duplicated genes is shown in Fig. 4A. The cis-element index of segmental and transposed duplicated genes ranged from 0.55 to 0.85 and 0.54 to 0.8 , respectively, demonstrating that wide divergences occurred in the promoter regions of duplicated gene pairs.

The cis-element types were summarized. The top 30 cis-element types are shown in Fig. 4B. Function annotations of these cis-elements were related to hormone regulations, cellular development, and stress response. For hormone regulation, the functions of cis-elements were mainly involved in responses related to salicylic acid, auxin, and abscisic acid metabolism. Cellular development mainly included meristem-specific activation, endosperm expression, palisade mesophyll cells, leaf morphology development, shoot-specific expression, and cell-cycle regulation. The remaining cis-elements were related to stress physiology, such as responses to light, low-temperature, heat, drought, dehydration, anaerobic induction, wound, and anoxic-
Fig. 2. The positions of $C l R 2 R 3-M Y B$ genes in the watermelon genome and duplication events. Blue lines indicate transposed duplication gene pairs. Orange lines indicate segmental duplication gene pairs. Red line represents the tandem duplication gene pair. Green line represents the proximal duplication gene pair. $\mathrm{Chr}=$ chromosome. 


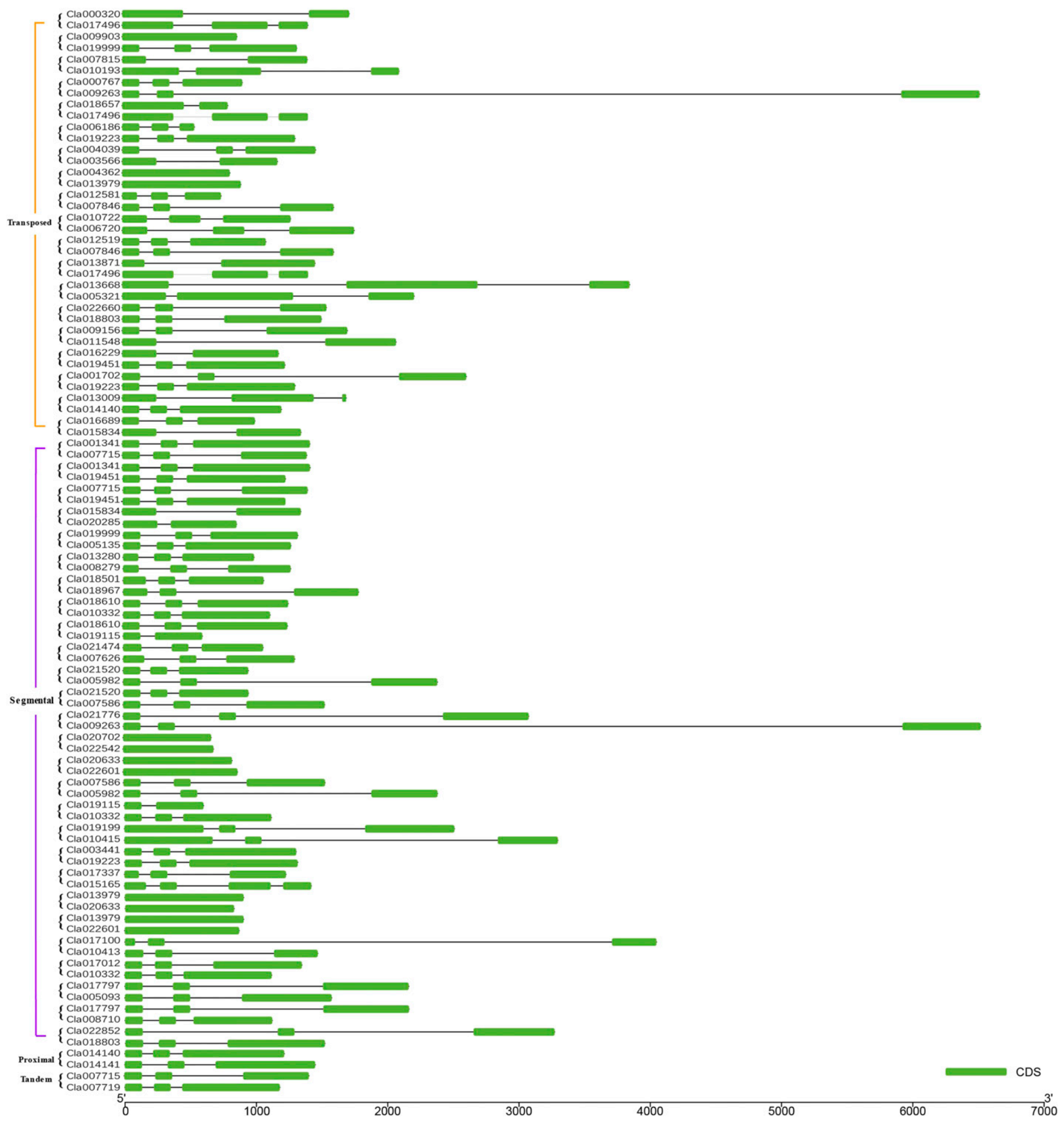

Fig. 3. Gene structures of duplicated $R 2 R 3-M Y B$ gene pairs. Green boxes represent the coding sequence (CDS) regions. Black lines show the intron regions. Yellow indicates transposed duplicated gene pairs. Purple indicates segmental duplicated gene pairs. The proximal and tandem duplicated gene pairs each have a pair of duplicated genes.

specific inducibility. The cis-element sequences CAAT-box and TATA-box were found in all ClR2R3-MYB genes. The light response of cis-elements Box-I, Box-4, and GAG-motif occurred in the promoter regions of most duplicated ClR2R3$M Y B$ s. A total of 59 duplicated $C l R 2 R 3-M Y B$ genes had the ARE cis-acting regulatory element, which is essential for anaerobic induction. Skn-1motif was found in 62 duplicated genes, suggesting these genes are putatively involved in the regulation of seed development. The 5UTRPy-rich stretch and G-box were also identified in more than $50 \mathrm{ClR} 2 R 3-M Y B$ promoter sequences, suggesting that these genes probably have high expression levels. TC-rich repeats, which are involved in defense and the stress response, were also found in the promotor regions of $54 C l R 2 R 3-M Y B$ genes. 

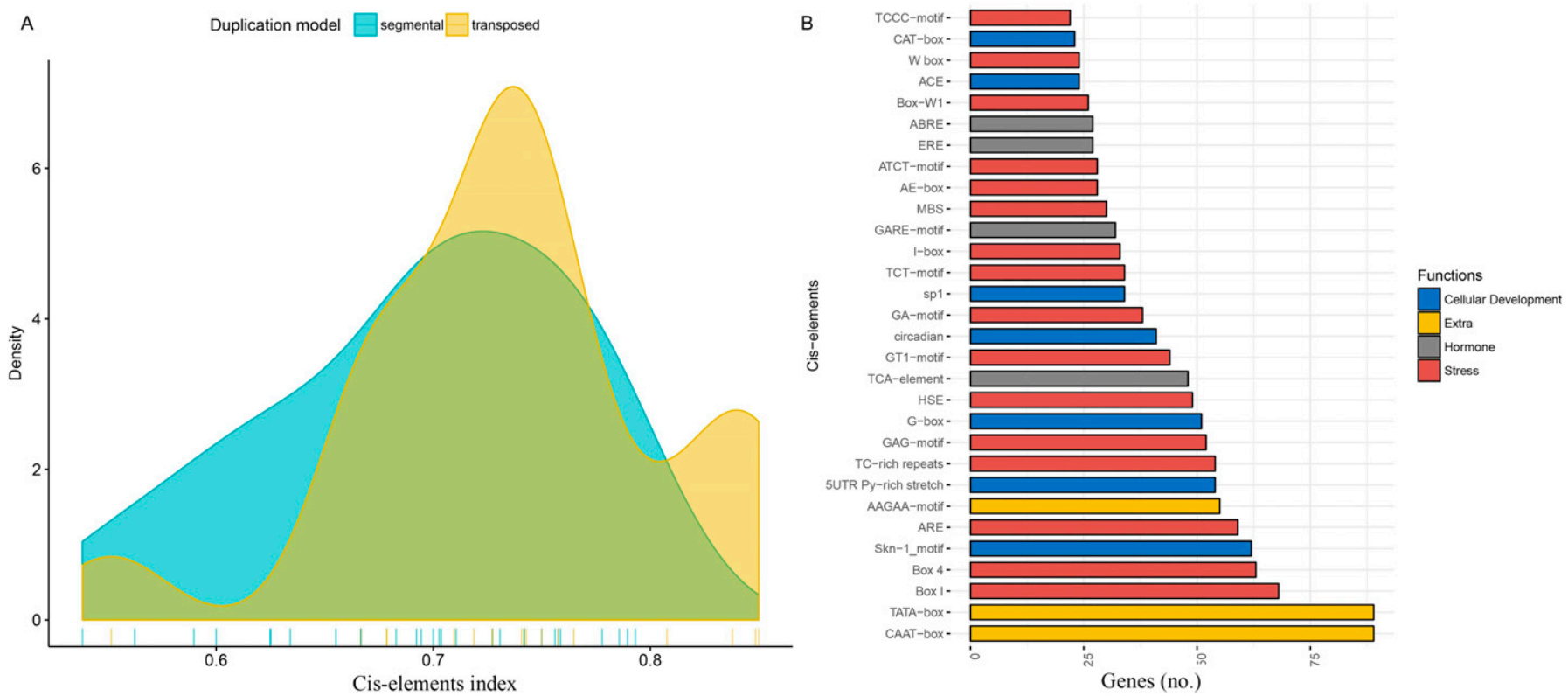

Fig. 4. The cis-element summary and density distribution of the cis-element index. (A) The cis-elements index density of duplicated gene pairs. (B) The cis-element summary. Annotations of cis-elements are listed in Supplemental Table 3.

Ka ANd Ks Substitution rates of duplicated CLR2R3MYB GENES. Ka, Ks, and their ratios were calculated. The density distribution of Ks values is presented in Fig. 5A. The Ks values ranged from 0.42 to 3.95 . The smallest Ks value was observed in the tandem duplicated gene pair Cla014140Cla014141. A peak appeared at $\approx 2.8$ in the density distribution plot, indicating that the duplication events occurred at one major stage and varied with the time of evolutionary process.

$\mathrm{The} \mathrm{Ka} / \mathrm{Ks}$ value is an index estimating the selection pressure during the gene evolution process. The $\mathrm{Ka} / \mathrm{Ks}$ values of the $R 2 R 3-M Y B$ duplicated gene pairs ranged from 0.06 to 0.32 , with an average of 0.15 (Fig. 5B). All the $\mathrm{Ka} / \mathrm{Ks}$ values were less than 1.00, suggesting that $C l R 2 R 3-M Y B$ duplicated genes had undergone purifying selection during the evolution process. Gene pairs with different duplication models tended to have different $\mathrm{Ka} / \mathrm{Ks}$ value distributions. The segmental duplicated gene pairs had relatively low $\mathrm{Ka} / \mathrm{Ks}$ values, whereas the transposed duplicated gene pairs displayed relatively high $\mathrm{Ka} /$ Ks values. A maximum $\mathrm{Ka} / \mathrm{Ks}$ value of 0.32 occurred in the tandem duplicated gene pair Cla014140-Cla014141, suggesting that the two genes might experience more relaxed purifying selection.

EXPRESSION DIVERGENCES OF DUPLICATED CLR2R3-MYB GENES. RNA-seq data derived from the fleshes and rinds of watermelon fruit at 10,18,26, and $34 \mathrm{DAP}$ and from seeds at $25,31,37$, and 49 DAP were used to determine the expression profiles of $C l R 2 R 3-M Y B$ family genes. A total of 34 genes were not expressed during watermelon fruit development. The remaining 55 genes were involved in the regulation of fruit development and maturity. Among them, 10 genes including Cla006761, Cla021243, Cla011548, Cla013099, Cla007114, Cla020633, Cla013668, Cla022601, Cla010413, and Cla013979 were constitutively expressed in all tissues and at all developmental stages. However, some ClR2R3-MYB genes exhibited tissue-specific expression patterns. Twelve ClR2R3MYB genes including Cla007815, Cla007846, Cla015834, Cla013461, Cla018610, Cla021474, Cla018967, Cla010415,
Cla017337, Cla017267, Cla017012, and Cla016980 were only expressed in seeds. Cla021520, Cla019115, and Cla017797 were specifically expressed in fleshes, whereas Cla005982, Cla008278, Cla019115, and Cla021520 were specifically expressed in the rinds.

To compare expression patterns of the duplicated genes, pairwise transcript abundances were illustrated in the heatmap (Fig. 6). A total of eight duplicated gene pairs were not expressed during watermelon fruit development, including one tandem duplicated gene pair, one proximal duplicated gene pair, two transposed duplicated pairs, and four segmental duplicated gene pairs. Three segmental duplicated gene pairs of Cla013979-Cla022601, Cla020633-Cla022601, and Cla013979-Cla020633 were expressed in all tissues and at all stages of watermelon fruit development. To further quantify the expression similarity of duplicated gene pairs, Pearson's correlation coefficient was calculated for each expressed duplicated gene pair during watermelon fruit development. The significant correlation coefficients were marked aside the corresponding gene pairs $(P<0.05)$. A transposed duplicated gene pair of Cla004362-Cla013979 and a segmental duplicated gene pair of Cla020702-Cla022542 exhibited significantly similar expression patterns, demonstrating that the duplicated gene pairs had putatively similar functions during watermelon fruit development. Although the expression patterns were significantly correlated, significantly different transcript abundances were also observed between the two duplicated gene pairs. The remaining 38 duplicated gene pairs exhibited divergent expression patterns, suggesting different regulatory roles during fruit development.

Tissue-specific divergent expression patterns were observed between duplicated gene pairs. Five transposed duplicated gene pairs exhibited tissue-specific divergent expression patterns. Cla019999 was expressed in rind and flesh, but the expression of its duplicated gene Cla009903 was not detected in the two tissues. Cla009263 was expressed in all tissues, but Cla000767 was not expressed in the fruit. Similarly, Cla015834 was 

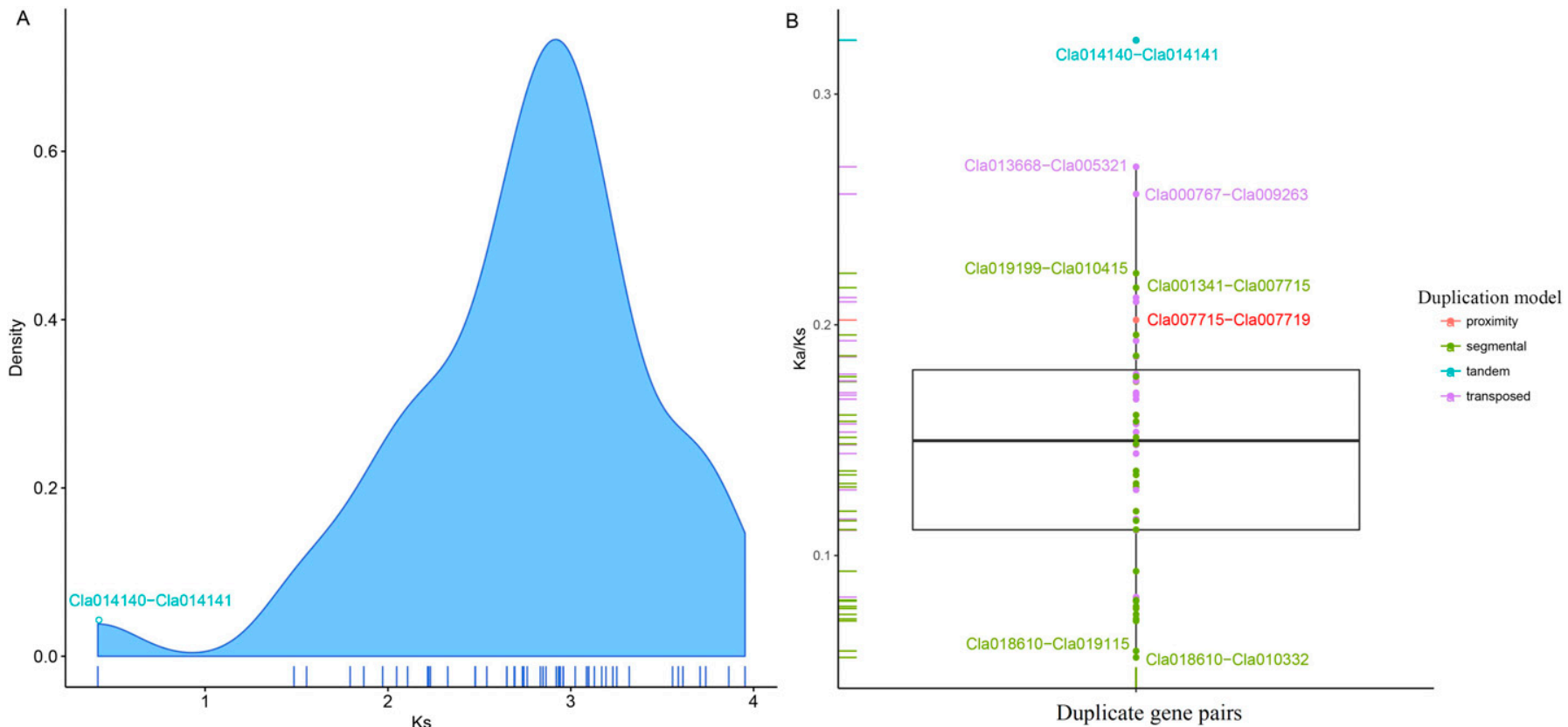

Fig. 5. Density distribution of synonymous mutation (Ks) and boxplot of nonsynonymous mutation (Ka)/Ks values: (A) density distribution of Ks for the $C l R 2 R 3$ $M Y B$ duplicated gene pairs and (B) $\mathrm{Ka} / \mathrm{Ks}$ value distribution of $C l R 2 R 3-M Y B$ duplicated gene pairs.

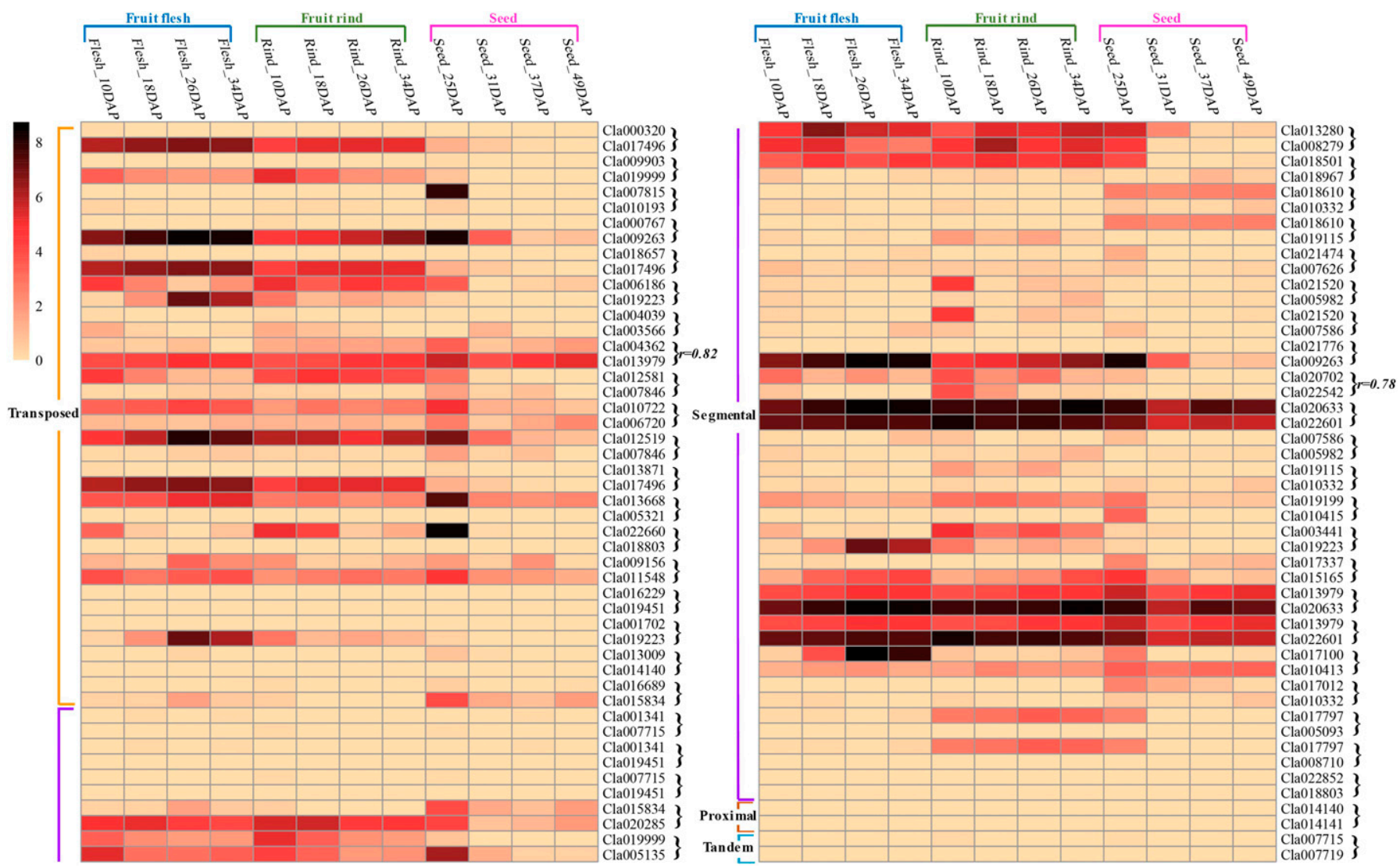

Fig. 6. Expression profiles of duplicated $C l R 2 R 3-M Y B$ gene pairs in seed, fruit flesh, and rind during watermelon fruit development. The color scale at the left of each dendrogram represents $\log 2$ expression values, with black indicating high levels and white indicating low levels of transcript abundance. $r=$ correlation coefficient expression of two duplicated genes. 
specifically expressed in the seed, but its duplicated gene Cla016689 was not expressed in the fruit. Cla019223 was expressed in rind and flesh, but its duplicated gene Cla001702 was not expressed in the fruit. Cla013668 was expressed in all tissues and at all stages, but expression of its duplicated gene Cla005321 was not detected in the fruit. Tissue-specific divergent expression patterns were also found in the four segmental duplicated gene pairs. Cla019115 was only expressed in the rind, but its duplicated gene Cla010332 was not expressed in the fruit. Cla018501 was expressed in all tissues, whereas its duplicated gene Cla018967 was only expressed in the seed. Cla018610 was only expressed in the seed, but its duplicated gene Cla019115 was only expressed in the rind. Cla018610 was only expressed in the seed, but its duplicated gene Cla010332 was not expressed in the fruit.

Development stage-specific divergent expression patterns were also observed between duplicated gene pairs. Regarding transposed duplicated gene pairs, Cla018657 was not expressed in fruit, but its duplicated gene $\mathrm{Cla} 017496$ was expressed in rinds and fleshes at all stages, as well as in the seed at 10 DAP. Cla012519 was expressed in all tissues and at all stages, but its duplicated gene Cla007846 was only expressed in the seed at 25 and 31 DAP. Cla007815 was only expressed in seed at 25 DAP, whereas its duplicated gene Cla010193 was not expressed in fruit. Similar results were also observed in segmental duplicated gene pairs. For instance, Cla020702 was expressed in all tissues, but its duplicated gene Cla022542 was only expressed in the rinds at 10 and 18 DAP. Similarly, Cla017337 was only expressed in the seed at 25 and 49 DAP, but its duplicated gene Cla015165 was expressed in all tissues. Cla019199 was expressed in all tissues but not in the seed at 25 DAP, whereas its duplicated gene Cla010415 was only expressed in the seed at 25 DAP. Cla003441 was expressed in the rind and flesh at 10 DAP. However, Cla019223 was only expressed in the rind and flesh at 18, 26, and 34 DAP.

\section{Discussion}

$R 2 R 3-M Y B$ transcription factors are widely involved in the regulations of plant morphogenesis, growth, metabolism, developmental processes, and response to biotic or abiotic stresses. However, duplication types of watermelon $R 2 R 3-$ $M Y B$ and their possible roles regulating watermelon fruit development have been ignored. In this study, the duplicated ClR2R3-MYB genes were identified, and their expression profiles were investigated during fruit development to understand their potential roles during watermelon fruit development.

A total of $89 R 2 R 3-M Y B$ genes were identified in the watermelon genome; this number was similar to that of previous reports (Xu et al., 2018) but larger than that reported for cucumber (Cucumis sativus) (Ren et al., 2009) and smaller than that identified in Arabidopsis thaliana (Stracke et al., 2001). All ClR2R3-MYBs have two incomplete R. Every R forms an HTH structure, which is consistent with $R 2 R 3-M Y B$ family characteristics identified in the present study. The first Trp residue in the R3 repeat was replaced by some hydrophobic amino acids like Phe or Ile. Similar results were also observed in A. thaliana (Stracke et al., 2001) and cucumber (Li et al., 2012). However, some other residues, such as Glu, Gly, Lys, Ser, Cys, Leu, Thr, Asp, and Asn, were specifically conserved in watermelon $R 2 R 3-M Y B$ family genes.

A total of $48 C l R 2 R 3-M Y B$ duplicated gene pairs were identified in this study. Segmental duplication accounted for
$56.25 \%$ of the total duplication events, demonstrating that it has a major role in ClR2R3-MYB gene expansion. Similar results were also reported for pineapple (Liu et al., 2017). Results of the present study suggested that genes produced by segmental duplications were retained during evolution (Cannon et al., 2004). In addition, mutually segmental duplicated events were found among the triple genes of Cla013979-Cla020663-Cla022601, Cla005982-Cla007586Cla021520, Cla010332-Cla019115-Cla018610, and Cla007715-Cla019451-Cla001341, which probably occurred along with the triplicate evolution of the watermelon genome (Guo et al., 2013). One proximal gene pair and one tandem gene pair were detected in ClR2R3-MYBs. Low frequencies of tandem and proximity duplicated gene pairs in the $R 2 R 3-M Y B$ family were also found in $A$. thaliana (Cannon et al., 2004). Transposed duplication accounted for $41.7 \%$ of the total duplication events, which also had an important role in the expansion of $C l R 2 R 3-$ $M Y B$ genes. Similar results were also reported for family genes related to sorbitol metabolism pathways (Qiao et al., 2018).

Different cis-regulatory elements in the promoter sequences of duplicated genes may produce different expression patterns. In this study, most duplicated gene pairs had divergent regulatory elements. There were four transposed duplicated gene pairs with a cis-element index more than 0.8 , suggesting that their expressions can be regulated by several different transacting factors (Qiao et al., 2018). Several studies have shown that the duplicated genes inherited the transcription regulatory models from their ancestors, but that their cis-regulatory regions were divergent after evolution (Haberer et al., 2004). The highly divergent cis-regulatory elements between $C l R 2 R 3$ $M Y B$ gene pairs potentially lead to sub-functionalization or neo-functionalization between the duplicated genes.

Expression patterns of $C l R 2 R 3-M Y B$ duplicated genes were measured in flesh, rind, and seed during watermelon fruit development. Only two duplicated gene pairs, Cla004362Cla013979 and Cla020702-Cla022542, had similar expression patterns, whereas less nonsynonymous mutations, similar gene structures, and cis-element compositions were observed. This suggested that function redundancy or sub-functionalization probably occurred between the two genes. Similar results were also observed between GmPIN duplicated genes in soybean (Glycine max) (Liu and Wei, 2017). The other 38 duplicated gene pairs exhibited tissue-specific or development stagespecific divergent expression patterns during watermelon fruit development. Different gene structures and cis-element compositions, as well as relatively high nonsynonymous mutations, were observed between these duplicated pairs, suggesting that neo-functionalization probably occurred after duplication. Similar results were also reported between the segmental duplication of $M Y B$ homologous genes in maize (Zea mays) (Zhang et al., 2000). Neo-functionalization potentially leads to the importance of $C l R 2 R 3-M Y B$ duplicated genes in the regulation of watermelon fruit development.

\section{Literature Cited}

Bray, N.L., H. Pimentel, P. Melsted, and L. Pachter. 2016. Nearoptimal probabilistic RNA-seq quantification. Nat. Biotechnol. 34:525-527.

Cannon, S.B., A. Mitra, A. Baumgarten, N.D. Young, and G. May. 2004. The roles of segmental and tandem gene duplication in the evolution of large gene families in Arabidopsis thaliana. BMC Plant Biol. 4:10. 
Crooks, G.E., G.C. Hon, J.M. Chandonia, and S.E. Brenner. 2004 WebLogo: A sequence logo generator. Genome Res. 14:1188-1190. D’Amelia, V., R. Aversano, A. Ruggiero, G. Batelli, I. Appelhagen, C. Dinacci, L. Hill, C. Martin, and D. Carputo. 2018. Subfunctionalization of duplicated MYB genes in Solanum commersonii generated the cold-induced $S c A N 2$ and the anthocyanin regulator $S c A N 1$. Plant Cell Environ. 41:1038-1051.

Duarte, J.M., L. Cui, P. Kerr Wall, Q. Zhang, X. Zhang, J. LeebensMack, H. Ma, N. Altman, and C. dePamphilis. 2006. Expression pattern shifts following duplication indicative of subfunctionalization and neofunctionalization in regulatory genes of Arabidopsis. Mol. Biol. Evol. 23:469-478.

Dubos, C., R. Stracke, E. Grotewold, B. Weisshaar, C. Martin, and L. Lepiniec. 2010. MYB transcription factors in Arabidopsis. Trends Plant Sci. 15:573-581.

El-Gebali, S., J. Mistry, A. Bateman, S.R. Eddy, A. Luciani, S.C. Potter, M. Qureshi, L.J. Richardson, G.A. Salazar, A. Smart, E.L.L. Sonnhammer, L. Hirsh, L. Paladin, D. Piovesan, S.C.E. Tosatto, and R.D. Finn. 2019. The Pfam protein families database in 2019. Nucleic Acids Res. 47:D427-D432.

Finn, R.D., J. Clements, and S.R. Eddy. 2011. HMMER web server: Interactive sequence similarity searching. Nucleic Acids Res. 39:W29-W37.

Flagel, L.E. and J.F. Wendel. 2009. Gene duplication and evolutionary novelty in plants. New Phytol. 183:557-564.

Food and Agriculture Organization of the United Nations. 2018. FAOSTAT crops data. 8 June 2019. <http://www.fao.org/home/en>. Freeling, M. 2009. Bias in plant gene content following different sorts of duplication: Tandem, whole-genome, segmental, or by transposition. Annu. Rev. Plant Biol. 60:433-453.

Freeling, M., M.J. Scanlon, and J.E. Fowler. 2015. Fractionation and subfunctionalization following genome duplications: Mechanisms that drive gene content and their consequences. Curr. Opin. Genet. Dev. 35:110-118.

Guo, S., J. Zhang, H. Sun, J. Salse, W.J. Lucas, H. Zhang, Y. Zheng, L. Mao, Y. Ren, and Z. Wang. 2013. The draft genome of watermelon (Citrullus lanatus) and resequencing of 20 diverse accessions. Nat. Genet. 45:51-58.

Haberer, G., T. Hindemitt, B.C. Meyers, and K.F.X. Mayer. 2004. Transcriptional similarities, dissimilarities, and conservation of ciselements in duplicated genes of Arabidopsis. Plant Phytol. 136:3009-3022.

Hahn, M.W. 2009. Distinguishing among evolutionary models for the maintenance of gene duplicates. J. Hered. 100:605-617.

Hu, B., J. Jin, A. Guo, H. Zhang, J. Luo, and G. Gao. 2015. GSDS 2.0: An upgraded gene feature visualization server. Bioinformatics 31:1296-1297.

Jia, L., M.T. Clegg, and T. Jiang. 2004. Evolutionary dynamics of the DNA-binding domains in putative $R 2 R 3-M Y B$ genes identified from rice subspecies indica and japonica genomes. Plant Phytol. 134:575585 .

Krzywinski, M., J.E. Schein, I. Birol, J.M. Connors, R.D. Gascoyne, D. Horsman, S.J.M. Jones, and M.A. Marra. 2009. Circos: An information aesthetic for comparative genomics. Genome Res. 19:1639-1645.

Lescot, M., P. Dehais, G. Thijs, K. Marchal, Y. Moreau, Y.V. De Peer, P. Rouze, and S. Rombauts. 2002. PlantCARE, a database of plant cis-acting regulatory elements and a portal to tools for in silico analysis of promoter sequences. Nucleic Acids Res. 30:325-327.

Letunic, I. and P. Bork. 2018. 20 years of the SMART protein domain annotation resource. Nucleic Acids Res. 46:D493-D496.

Li, Q., C. Zhang, J. Li, L. Wang, and Z. Ren. 2012. Genome-wide identification and characterization of $R 2 R 3-M Y B$ family in Cucumis sativus. PLoS One 7:e47576, doi: 10.1371/journal.pone.0047576.

Liu, C., T. Xie, C. Chen, A. Luan, J. Long, C. Li, Y. Ding, and Y. He. 2017. Genome-wide organization and expression profiling of the $R 2 R 3-M Y B$ transcription factor family in pineapple (Ananas comosus). BMC Genomics 18:503.
Liu, Y. and H. Wei. 2017. Genome-wide identification and evolution of the PIN-FORMED (PIN) gene family in Glycine max. Genome 60:564-571.

Long, M. and K.R. Thornton. 2001. Gene duplication and evolution. Science 293(5535): 1551.

Machemer, K., O. Shaiman, Y. Salts, S. Shabtai, I. Sobolev, E. Belausov, E. Grotewold, and R. Barg. 2011. Interplay of $M Y B$ factors in differential cell expansion, and consequences for tomato fruit development. Plant J. 68:337-350.

Panchy, N., M.D. Lehti-Shiu, and S. Shiu. 2016. Evolution of gene duplication in plants. Plant Phytol. 171:2294-2316.

Qiao, X., H. Yin, L. Li, R. Wang, J. Wu, J. Wu, and S. Zhang. 2018. Different modes of gene duplication show divergent evolutionary patterns and contribute differently to the expansion of gene families involved in important fruit traits in pear (Pyrus bretschneideri). Front. Plant Sci. 9:161.

Quinlan, A.R. and I.M. Hall. 2010. BEDTools: A flexible suite of utilities for comparing genomic features. Bioinformatics 26:841.

Ren, Y., Z. Zhang, J. Liu, J.E. Staub, Y. Han, Z. Cheng, X. Li, J. Lu, H. Miao, H. Kang, B. Xie, X. Gu, X. Wang, Y. Du, W. Jin, and S. Huang. 2009. An integrated genetic and cytogenetic map of the cucumber genome. PLoS One 4:e5795, doi: 10.1371/journal.pone.0005795.

Rosinski, J.A. and W.R. Atchley. 1998. Molecular evolution of the Myb family of transcription factors: Evidence for polyphyletic origin. J. Mol. Evol. 46:74-83.

Salman-Minkov, A., N. Sabath, and I. Mayrose. 2016. Whole-genome duplication as a key factor in crop domestication. Nat. Plants 2:16115.

Stracke, R., M. Werber, and B. Weisshaar. 2001. The R2R3-MYB gene family in Arabidopsis thaliana. Curr. Opin. Plant Biol. 4:447-456.

Wang, Y., J. Li, and A.H. Paterson. 2013a. MCScanX-transposed: Detecting transposed gene duplications based on multiple colinearity scans. Bioinformatics 29:1458-1460.

Wang, Y., X. Tan, and A.H. Paterson. 2013b. Different patterns of gene structure divergence following gene duplication in Arabidopsis. BMC Genomics 14:652.

Wang, Y., H. Tang, J.D. Debarry, X. Tan, J. Li, X. Wang, T. Lee, H. Jin, B.S. Marler, and H. Guo. 2012. MCScanX: A toolkit for detection and evolutionary analysis of gene synteny and collinearity. Nucleic Acids Res. 40:e49, doi: 10.1093/nar/gkr1293.

Waterhouse, A., M. Bertoni, S. Bienert, G. Studer, G. Tauriello, R. Gumienny, F.T. Heer, T.A.P. De Beer, C. Rempfer, L. Bordoli, R. Lepore, and T. Schwede. 2018. SWISS-MODEL: Homology modelling of protein structures and complexes. Nucleic Acids Res. 46:W296-W303.

Xu, Q., J. He, J. Dong, X. Hou, and X. Zhang. 2018. Genomic survey and expression profiling of the $M Y B$ gene family in watermelon. Hort. Plant J. 4:5-19.

Zhang, P., S. Chopra, and T. Peterson. 2000. A segmental gene duplication generated differentially expressed myb-homologous genes in maize. Plant Cell 12:2311-2322.

Zhang, S., J. Zhang, J. Zhao, and C. He. 2015. Distinct subfunctionalization and neofunctionalization of the B-class MADS-box genes in Physalis floridana. Planta 241:387-402.

Zhang, Z., J. Li, X. Zhao, J. Wang, G. Wong, and J. Yu. 2006. KaKs_Calculator: Calculating $\mathrm{Ka}$ and $\mathrm{Ks}$ through model selection and model averaging. Genom. Proteom. Bioinfo. 4:259-263.

Zheng, Y., Y. Xu, Y. Weng, M. Mazourek, U.K. Reddy, K. Ando, J.D. Mccreight, A.A. Schaffer, J. Burger, Y. Tadmor, N. Katzir, X. Tang, Y. Liu, J.J. Giovannoni, K. Ling, W.P. Wechter, A. Levi, J. Garciamas, R. Grumet, and Z. Fei. 2019. Cucurbit Genomics Database $(\mathrm{CuGenDB})$ : A central portal for comparative and functional genomics of cucurbit crops. Nucleic Acids Res. 47:D1128-D1136.

Zhu, Y., N. Wu, W. Song, G. Yin, Y. Qin, Y. Yan, and Y. Hu. 2014. Soybean (Glycine max) expansion gene superfamily origins: Segmental and tandem duplication events followed by divergent selection among subfamilies. BMC Plant Biol. 14:93. 
Supplemental Table 1. Characteristics of watermelon $R 2 R 3-M Y B$ genes.

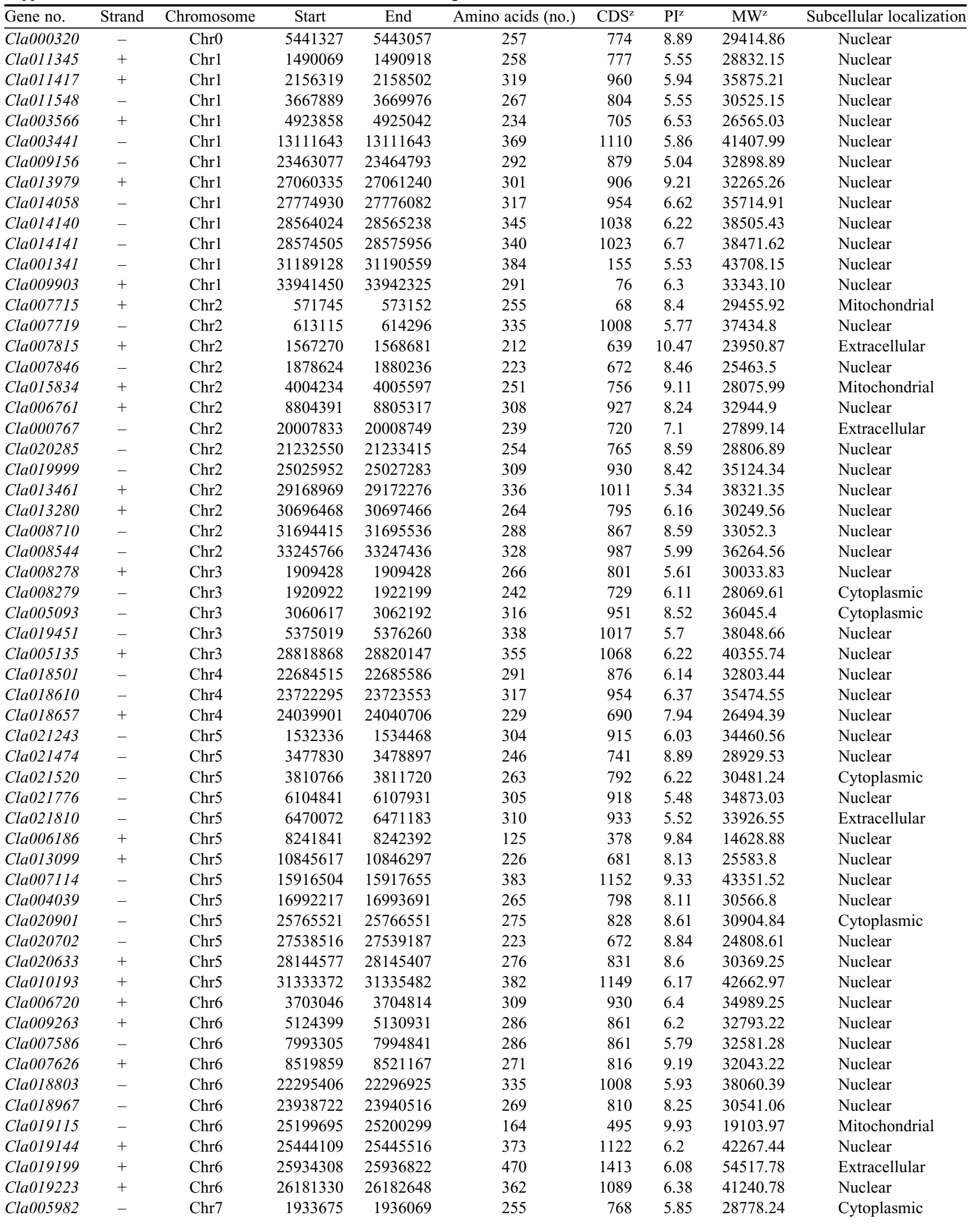

Continued next page 
Supplemental Table 1. Continued.

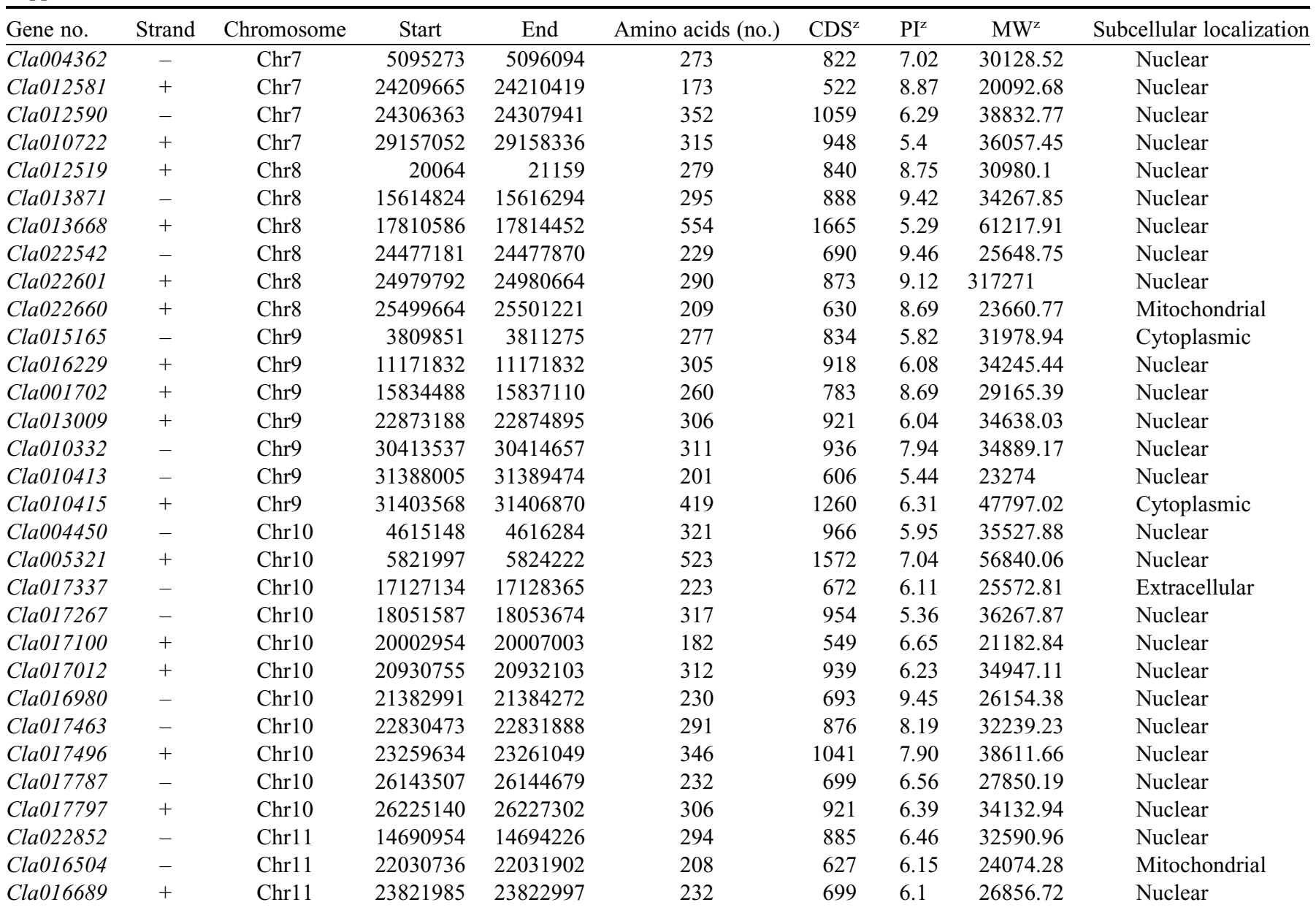

${ }^{\bar{z}} \mathrm{CDS}=$ coding sequence, $\mathrm{PI}=$ theoretical isoelectric point, $\mathrm{MW}=$ molecular weight. 
Supplemental Table 2. Duplication information of $R 2 R 3-M Y B$ gene pairs in watermelon.

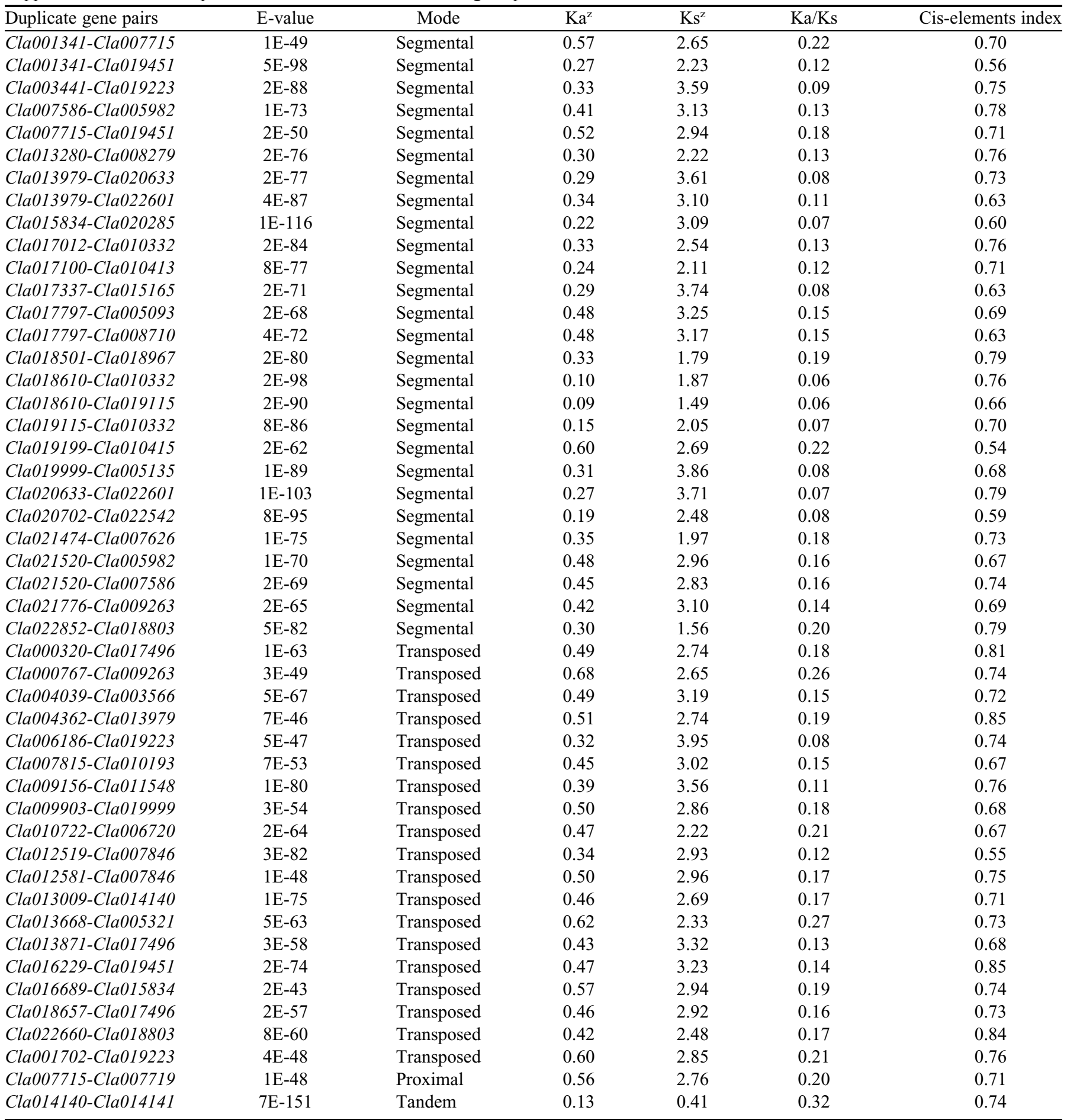

${ }^{\mathrm{z}} \mathrm{Ka}=$ synonymous mutation, $\mathrm{Ks}=$ nonsynonymous mutation. 
Supplemental Table 3. Cis-regulatory elements annotations.

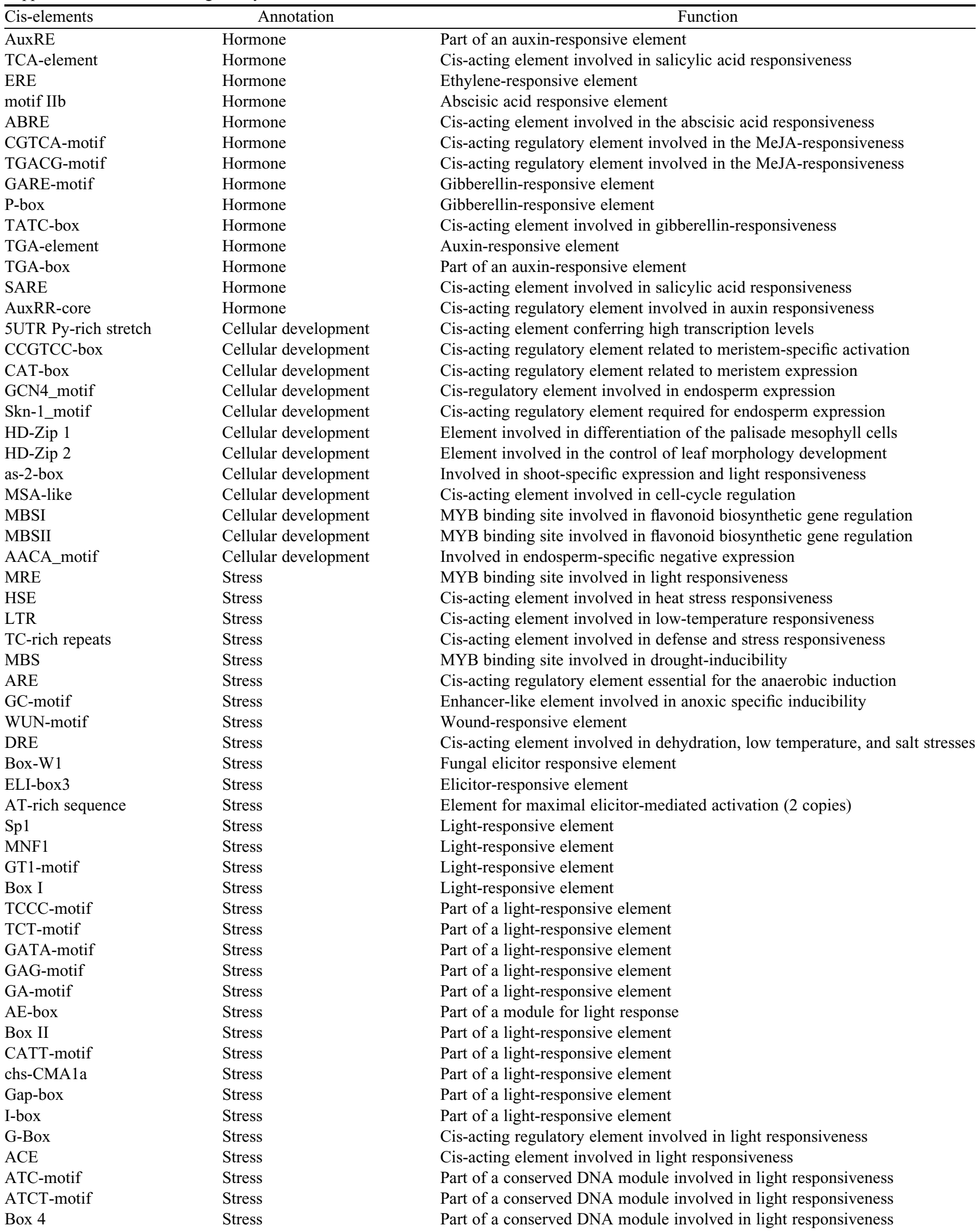


Supplemental Table 3. Continued.

\begin{tabular}{lll}
\hline Cis-elements & \multicolumn{1}{c}{ Annotation } & Function \\
\hline 3-AF1 binding site & Stress & Light-responsive element \\
AAAC-motif & Stress & Light-responsive element \\
4cl-CMA2b & Stress & Light-responsive element \\
Pc-CMA2a & Stress & Part of a light-responsive element \\
AT1-motif & Stress & Part of a light-responsive module \\
L-box & Stress & Part of a light-responsive element \\
rbcS-CMA7a & Stress & Part of a light-responsive element \\
LAMP-element & Stress & Part of a light-responsive element \\
LS7 & Stress & Part of a light-responsive element \\
ATCC-motif & Stress & Part of a conserved DNA module involved in light responsiveness \\
H-box & Stress & Cis-acting regulatory element involved in light responsiveness \\
Box III & Stress & Protein binding site (involved in light responsiveness) \\
EIRE & Stress & Elicitor-responsive element \\
chs-Unit $1 \mathrm{~m} 1$ & Stress & Part of a light-responsive element \\
chs-CMA2a & Stress & Part of a light-responsive element \\
ACA-motif & Stress & Part of gapA in (gapA-CMA1) involved with light responsiveness \\
\hline
\end{tabular}

